\title{
La réaction acrosomique
}

\section{Revue bibliographique des techniques de détection et intérêt de l'exploration acrosomique}

\author{
S. PILIKIAN \\ Laboratoire de Biologie de la Reproduction et du Développement, Lyon
}

\section{RESUME}

La capacité des spermatozoïdes à accomplir la réaction acrosomique (RA) au contact de la membrane pellucide de l'ovocyte représente une fonction essentielle pour la réalisation de la fécondation. Plusieurs méthodes sont disponibles pour évaluer le statut acrosomique ; chaque méthode détecte une étape de la réaction qui est un processus continu. La multiplicité et la relative spécificité des différentes techniques rendent délicate la comparaison des résultats en valeur absolue.

La réaction est $\mathrm{Ca}^{++}$dépendante d'où l'intérêt pour l'utilisation des ionophores calciques. Ils permettent une étude dynamique qui donne une meilleure appréciation de la capacité des spermatozoïdes à réagir en présence d'un inducteur.

Compte tenu de l'importance de cette réaction pour la réussite de la fécondation, plusieurs équipes ont cherché à corréler le statut acrosomique avec le pouvoir fécondant des spermatozoïdes. Les résultats montrent que les spermatozoïdes hypofécondants manifestent une réponse diminuée à l'induction de la RA.

Des travaux systématiques avec un protocole bien défini semble nécessaires pour pouvoir comparer des résultats multicentriques et établir la valeur pronostique de cet examen.

Mots clés : Spermatozoides humains, tests de réaction acrosomique, réaction acrosomique, fécondation in vitro.

\section{INTRODUCTION}

La fonction principale du spermatozoïde est d'apporter son ADN à l'ovocyte. Pour cela il se sert à la fois de sa mobilité et de ses enzymes localisées dans la partie la plus antérieure de sa tête : L'ACROSOME.

L'acrosome est une vésicule aplatie entourée d'une membrane où l'on distingue la membrane acrosomique interne face à la membrane nucléaire et la membrane acrosomique externe face à la membrane plasmique du spermatozoïde. Les membranes acrosomiques interne et externe se joignent au niveau du segment équatorial. L'acrosome peut être assimilé à une vésicule sécrétoire ; les enzymes hydrolytiques qu'elle contient (acrosine, hyaluronidase, neuraminidase pour ne citer que les principales) sont libérćes par un phénomène d'exocytose suite à un signal approprié et participent vraisemblablement à la progression des gamètes mâles à travers les multiples enveloppes ovocytaires. L'activation et la libération sélectives de ces enzymes à mesure que les spermatozoïdes approchent l'ovocyte, sont contrôlées par les phénomènes complexes de capacitation et de réaction acrosomique, difficilement dissociables.

La nécessité pour les spermatozoïdes éjaculés de subir des modifications pour acquérir la capacité de féconder les ovocytes a été décrite pour la première fois en 1951 [1, 2]. Depuis, malgré le nombre considérable de travaux il subsiste encore une certaine confusion sur le sujet. Cela tient d'une part, aux conditions dans lesquelles ces phéno- 
mènes sont étudiés et qui ont un effet significatif sur la capacitation et la réaction acrosomique et d'autre part, aux différentes méthodes d'exploration, chaque méthode ne mettant en évidence qu'un aspect de ce processus continu. A cette complexité s'ajoutent les différences inter et intra spécifiques qui sont considérables.

Avec la maîtrise de la fécondation in vitro, un intérêt croissant est accordé à l'étude des différentes fonctions du gamète mâle fonction cinétique, fonction de capacitation et de réaction acrosomique, fonction fusiogène et fonction nucléaire- en relation avec la fécondation.

Nous aborderons la capacitation et la réaction acrosomique dans cette revue.

\section{La Capacitation : Condition nécessai- re à la réalisation de la réaction acro- somique}

Plusieurs composants des sécrétions testiculaires, épididymaires et du liquide séminal sont adsorbés à la surface des spermatozoïdes au cours de leur transit dans les voies génitales mâles et pendant l'éjaculation. Ces composants de nature glycoprotéique stabilisent la membrane spermatique en bloquant les récepteurs de surface. Le retrait de ces facteurs constitue la première étape de la capacitation qui peut être définie comme un conditionnement de la surface cellulaire [pour une revue bibliographique, voir les références 3 et 4]. Ainsi la membrane plasmique du spermatozoìde dans un milieu capacitant, se trouve dans un processus continu de remaniement de structure et de composition de la bicouche lipidique, à mesure qu'elle perd ces facteurs de surface.

Cette perte des molécules glycoprotéiques détectée par des Ac marqués et des lectines s'accompagne d'une réorganisation topographique de nombreuses glycoprotéines transmembranaires qui jouent le rôle de coupleur entre les récepteurs de surface et le cytosquelette [5]. Ces modifications peuvent être mises en évidence par la technique de cryofracture couplée à un marquage à l'or de ces protéines $[6,7,8]$.

Enfin cette réorganisation concerne également les protéines intra-membranaires : leurs liaisons avec la bicouche lipidique sont rompues [9] entraînant une certaine liberté de mouvement de ces protéines qui se déplacent et donnent naissance à des plages riches en particules protéiques et d'autres qui en sont dépourvues. C'est dans ces zones pauvres en protéines, plus perméables et plus instables, qu'auront lieu les fusions membranaires lors de la réaction acrosomique [10].

In vivo, les spermatozoïdes sont capacités au contact des sécrétions des voies génitales femelles pendant le transport des spermatozoïdes vers le site de fécondation. Pratiquement tous les agents qui captent le cholestérol ou qui augmentent les phospholipides membranaires, en diminuant le rapport cholestérol/phospholipides de la membrane plasmatique, entraînent la capacitation [11, 12]. En effet un rapport cholestérol/phospholipides proche de l'unité maintient la stabilité membranaire. Toute perturbation de ce rapport a pour conséquence une déstabilisation et une augmentation de la perméabilité de la bicouche lipidique.

Les facteurs qui "décapacitent" les spermatozoïdes adhérent à la surface du plasmalemme par des liaisons non-convalentes. In vitro, tous les traitements qui coupent ces liaisons ou qui hydrolysent ces facteurs ou encore altèrent la composition du glycocalyx induisent la capacitation. Ainsi les spermatozoïdes sont capacités après incubation dans un milieu hypertonique [13] ou contenant des glycosaminoglycanes, de l'héparine, des protéases ou des hydrolases $[14,15]$ ou encore de l'albumine [16].

\section{La Réaction Acrosomique (RA)}

Les spermatozoïdes de l'homme comme ceux de tous les mammiferes étudiés doivent effectuer la RA avant de pénétrer l'ovocyte. Du point de vue morphologique, cette réaction est caractérisée par la fusion ponctuelle des membranes plasmique et acroso- 
mique externe sous-jacente, qui donne lieu à la formation de vésicules constituées de ces 2 membranes. Ces vésicules se dispersent progressivement et le contenu de l'acrosome, hydrolysé par les enzymes acrosomiques, est libéré laissant exposée la membrane acrosomique interne.

On ne connaît pas exactement la chronologie des évènements moléculaires qui interviennent dans ce processus, ce qui, par contre, est certain, c'est la dépendance de cette réaction vis-à-vis du $\mathrm{Ca}^{++}$dont l'afflux intra-cellulaire déclenche une cascade de réactions complexes faisant intervenir des mécanismes d'activation et de transduction de signaux [17].

\section{a) Le rôle présumé $\mathrm{du} \mathrm{Ca}^{++}$peut se résu- mer comme suit :}

Le $\mathrm{Ca}^{++}$intra-cellulaire peut inactiver les ATP-ases membranaires avec pour conséquence une augmentation du $\mathrm{Na}^{+}$intra-cellulaire. Sous l'action d'un antiport $\mathrm{Na}^{+} / \mathrm{H}^{+}$, l'afflux des $\mathrm{H}^{+}$entraîne une élévation du $\mathrm{pH}$ intra-cellulaire qui activerait les enzymes acrosomiques [18].

En agissant avec les têtes polarisées des phospholipides membranaires, le $\mathrm{Ca}^{++}$peut contrebalancer les forces de répulsion électrostatiques entre les membranes plasmique et acrosomique externe et faciliter leur adhésion [19].

Le $\mathrm{Ca}^{++}$peut activer les phospholipases membranaires avec libération de lysophospholipides et d'acides gras fusiogènes [20].

- Enfin le $\mathrm{Ca}^{++}$peut agir comme un cofacteur et activer les récepteurs des protéines G. Ces protéines régulent l'activité d'enzymes comme l'adénylate cyclase et les phospholipases $\mathrm{A} 2$ et $\mathrm{C}$ qui entraînent la phosphorylation des protéines [21, 22, 23].

\section{b) Site naturel de la RA}

On sait que pour traverser la membrane pellucide, le spermatozoïde doit avoir effectué sa RA. Toutefois le lieu où elle se pro- duit a longtemps été un sujet de controverse. Cela est dû au fait que la réaction peut se produire spontanément en l'absence d'ovocyte, il suffit que les conditions pour sa réalisation soient réunies. Ainsi, les sécrétions du tractus génital femelle, riches en progestérone, prostaglandines et glycosaminoglycanes induisent la RA [24]. Cependant plusieurs études ont permis d'aboutir à la conclusion que chez les mammiferes, la RA du spermatozoïde fécondant se produit au contact de la membrane pellucide $[25,26$, 27], plus précisément, après reconnaissance spécifique et liaison avec la fraction glycoprotéique majeure, la ZP3 de $83 \mathrm{Kd}$, de cette membrane [28].

Le résultat de la réaction est :

- la libération de l'acrosine, dont le rôle présumé est de faciliter le passage du spermatozoïde à travers la membrane pellucide.

- l'extériorisation de la membrane acrosomique interne qui va permettre la reconnaissance spécifique de la membrane ovocytaire.

- la modification de la plaque équatoriale et de la cape post acrosomique dont le rôle est essentiel dans la fusion des 2 gamètes.

D'où l'importance accordée à l'étude de ce processus comme une des fonctions essentielles des gamètes mâles.

\section{Les différentes méthodes d'évalua- tion de la RA}

Il existe plusieurs techniques qui permettent d'apprécier le statut acrosomique :

- la microscopie électronique,

- les techniques d'immunofluorescence faisant appel à divers marqueurs comme les lectines et les Ac monoclonaux,

- les techniques histochimiques,

- l'étude de l'activité enzymatique.

La microscopie électronique est la méthode de référence qui a permis de décrire avec précision les différentes phases de la réaction et d'établir les critères d'une RA phy- 
siologique afin de la différencier d'une réaction dégénérative de l'acrosome après mort cellulaire.

Ces critères décrits par Russel et coll. [29] sont les suivants :

- absence de la membrane plasmique et de la membrane acrosomique externe dans la partie antérieure de la tête du spermatozoïde, avec présence ou absence des vésicules issues de la fusion de ces 2 membranes,

- intégrité de la membrane plasmique couvrant le segment équatorial,

- fusion de la membrane plasmique et de la membrane acrosomique externe dans la région équatoriale.

Cette méthode lourde et coûteuse ne peut être utilisée en routine, néanmoins elle doit servir de référence pour valider toute nouvelle méthode d'appréciation du statut acrosomique.

Le tableau de la page ci-contre illustre en résumé les caractéristiques des autres techniques, les sondes utilisées, le déterminant contre lequel ces sondes sont dirigées, leur localisation -membrane plasmique (MP), membrane acrosomique externe (MAE), matrice acrosomique, membrane acrosomique interne (MAI) - ainsi que les avantages et les inconvénients de chaque technique.

\section{Remarque sur les différentes méthodes}

La disponibilité de différentes techniques pour la mise en évidence de la RA a été en partie à l'origine de beaucoup de confusion et de contradiction rencontrées dans la littérature.

Compte tenu, d'une part, des caractéristiques de chaque méthode et d'autre part, des différentes phases de la réaction, il est évident qu'on n'aura pas exactement les mêmes résultats avec 2 techniques différentes. Par exemple, le pourcentage de RA relevé ne sera pas le même quand la sonde est dirigée contre la membrane plasmique ou contre la membrane acrosomique interne ; la lère détecte les modifications de la membrane plasmique qui ont lieu après la capacitation et au début de la réaction alors que la 2ème met en évidence la phase finale de la réaction, quand les vésicules sont dispersées et la membrane acrosomique interne est exposée.

La triple coloration a été très utilisée. Elle est peu coûteuse, nécessite un microscope à fond clair, permet de différencier une vraie réaction acrosomique d'une dégénérescence post-mortem de l'acrosome, les préparations sont permanentes. Cependant elle est longue et très sensible aux variations du $\mathrm{pH}$ des bains de colorant.

Les sondes fluorescentes sont de plus en plus utilisées. Il existe une variété de lectines conjuguées à la fluorescéine qui permettent une étude plus rapide de cette réaction. Toutefois il est capital de connaître la structure qu'elles reconnaissent et le signal émis en fluorescence (positif ou négatif) pour interpréter correctement des résultats et comparer à d'autres études. L'avantage de ces techniques est leur relative rapidité, elles sont peu onéreuses et peuvent être facilement couplées à un colorant vital fluorochrome (Hœchst 33258, propidium). Linconvénient majeur de ces méthodes est l'obtention de formes intermédiaires de fluorescence difficiles à classer.

Les Ac monoclonaux sont par contre plus spécifiques. Ils peuvent également être couplés à un colorant vital fluorochrome. Néanmoins, ces méthodes sont coûteuses et nécessitent un révélateur fluorescent de l'Ac (fluorescence indirecte) ce qui alourdit la technique.

Récemment, la préparation d'Ac fixés sur des billes magnétiques permet non seulement de détecter mais semble également offrir la possibilité de trier les spermatozoïdes réagis qui serviraient à la microinjection sous la zone pellucide (SUZI) [43, 44]. On pourrait ainsi réduire le nombre de 


\begin{tabular}{|c|c|c|c|c|c|c|c|}
\hline & 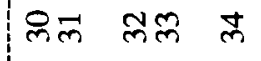 & చొ & $n^{\infty}$ & के & q & $F$ & F \\
\hline & 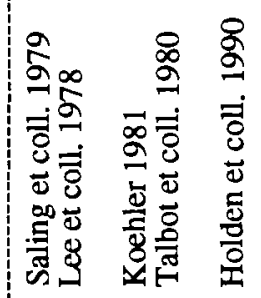 & 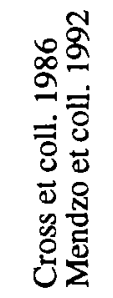 & 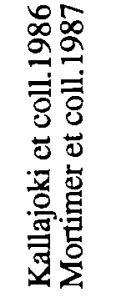 & 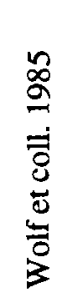 & 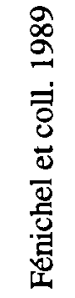 & 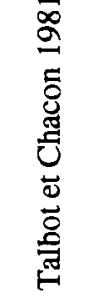 & 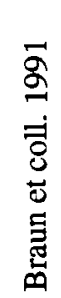 \\
\hline
\end{tabular}

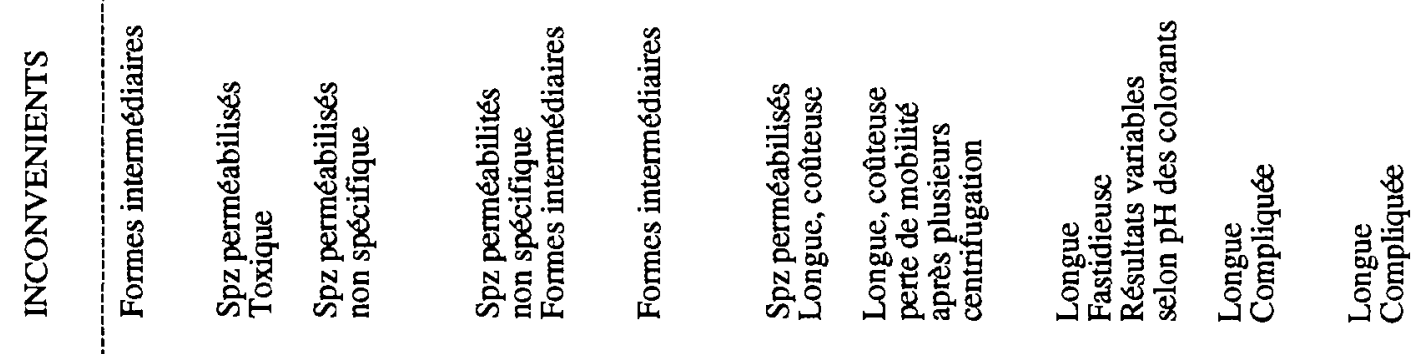

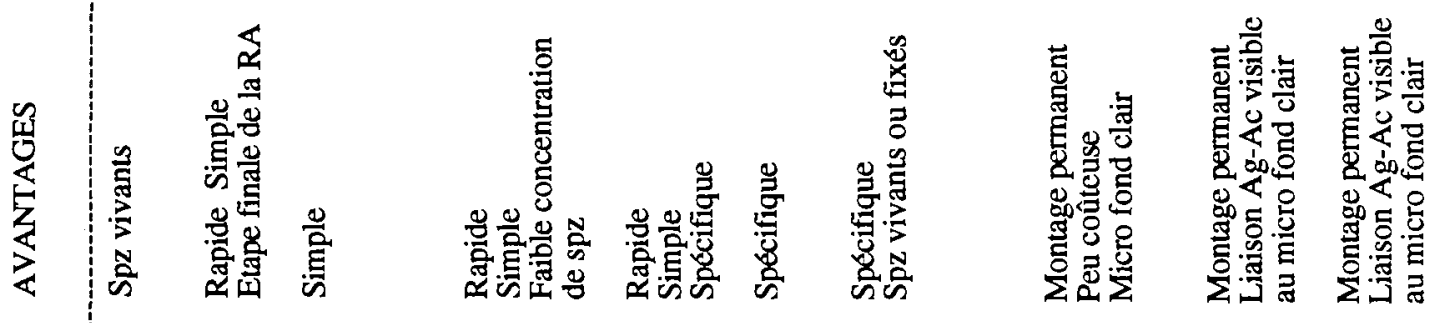

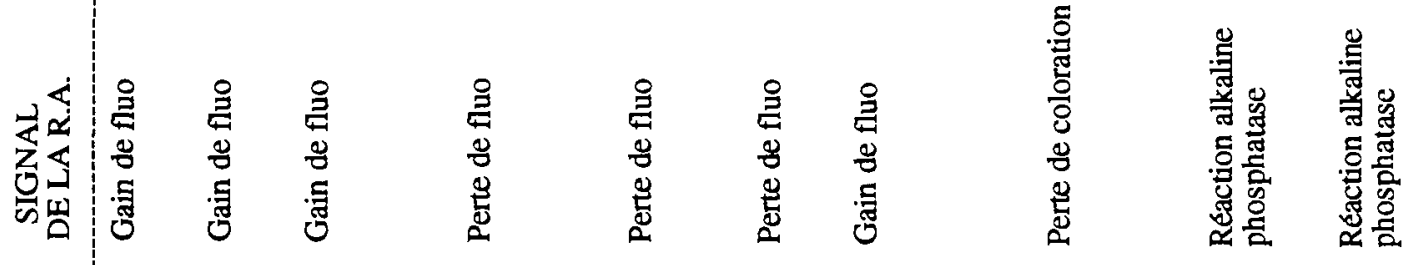

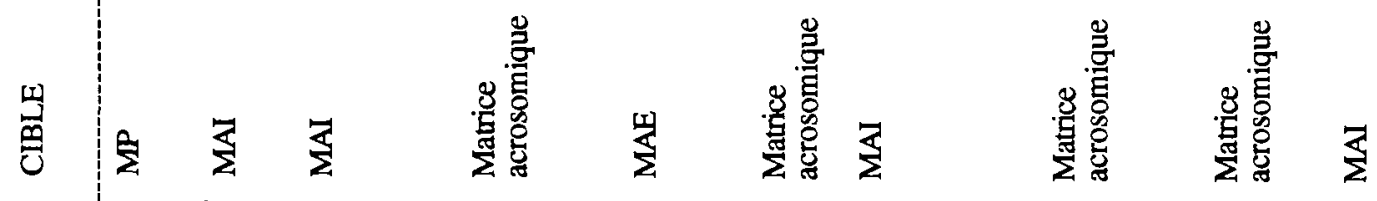

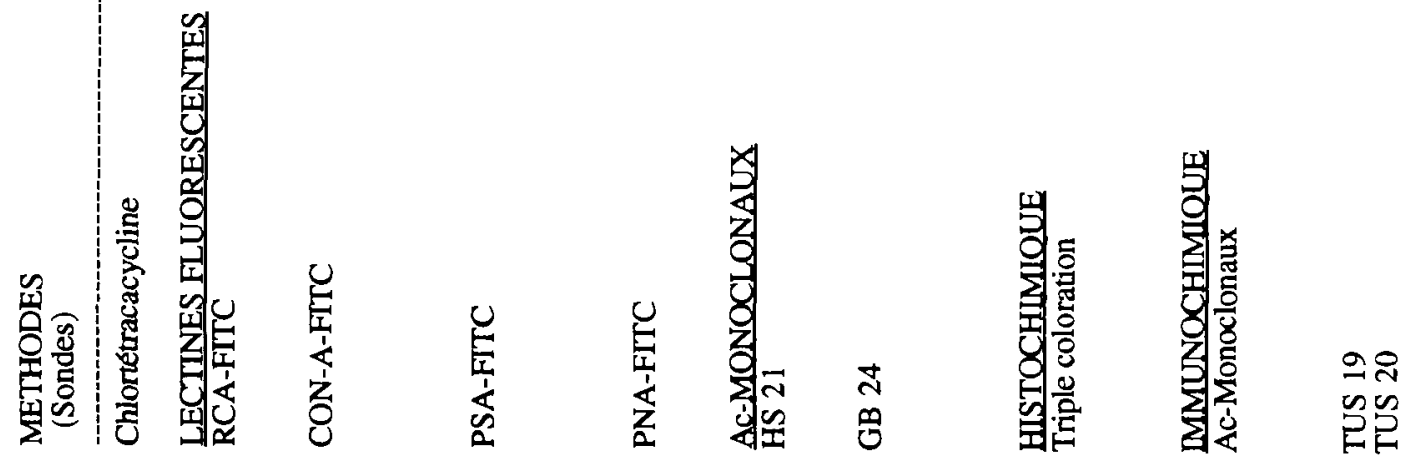


spermatozoïdes introduit dans l'espace périvitellin et éviter la polyspermie, tout en augmentant les chances d'une fécondation.

Enfin l'exploration du statut de l'acrosome peut être abordée par l'étude de l'activité de ses enzymes, essentiellement l'acrosine. Il existe plusieurs Ac monoclonaux susceptibles d'étudier sa localisation, sa répartition et son activité basée sur ses propriétés protéolytiques; dans ce cas il faut distinguer la pro-acrosine non-active, l'acrosine active et l'acrosine totale $[45,46,47]$. Des anomalies qualitatives et quantitatives de cette enzyme peuvent être à l'origine d'hypofécondité masculine [48, 49].

\section{INTERET ET CONDITIONS D'ETUDE DE LA RA}

La capacité des spermatozoïdes à réagir au contact de la membrane pellucide représente une fonction essentielle pour la réalisation de la fécondation. Par conséquent, l'évaluation de la RA dans le cadre d'une exploration d'une hypofécondité masculine paraît justifiée. Le problème est de définir les conditions de réalisation d'une telle étude pour attribuer à ce test une valeur pronostique.

Il est bien admis maintenant que le pourcentage de spermatozoïdes humains qui accomplit spontanément la RA dans les milieux classiques de FIV demeure faible (3 à 12\%) quels que soient le milieu et le temps d'incubation $[50,51,52,53]$. De plus, la réaction spontanée donne peu d'indication sur l'aptitude des spermatozoïdes à réagir au contact de la membrane pellucide, inducteur physiologique de la réaction du spermatozoïde qui va pénétrer l'ovocyte. Par conséquent une exploration dynamique, en présence d'un inducteur, semble plus appropriée.

\section{Quel inducteur choisir?}

Les inducteurs physiologiques, cellules du cumulus, liquide folliculaire ou membrane pellucide ne sont pas facilement disponibles. De plus, en raison d'une variabilité inhérente, les liquides biologiques ne sont pas les meilleurs candidats pour établir un protocole standardisé. Ainsi les résultats de réaction acrosomique varient en fonction de la concentration du liquide folliculaire dans le milieu de culture et en fonction de la teneur en progestérone du liquide folliculaire [54]. En l'absence, à ce jour, de recombinant de glycoprotéine ZP3 de la membrane pellucide, les ionophores calciques (A23187, ionomycine) sont utilisés, à titre expérimental, comme inducteurs in vitro. Sur le plan structural, la réaction induite par l'ionophore ne diffère pas de la réaction physiologique et le test est reproductible [29]. Les fortes concentrations de $\mathrm{A} 23187(50 \mathrm{\mu M})$, très toxiques, utilisées au début, entrâ̂naient une nécrospermie massive qui biaisait les résultats. Nous préconisons une concentration finale de $10 \mu \mathrm{M}$ dans le milieu de culture pour un effet positif significatif et une bonne survie [55].

Pour l'estimation du taux de R.A. il serait plus judicieux de comptabiliser uniquement les spermatozoïdes fraîchement réagis en réponse à l'induction $[56,57]$ seule cette réponse dynamique aurait une signification biologique car on ne connaît pas le devenir des spermatozoïdes prématurément réagis.

\section{Relation entre la RA et la fécondan- ce du spermatozoïde}

Compte tenu du rôle essentiel de l'acrosome dans la fécondation, la recherche d'une relation directe entre le statut acrosomique et le pouvoir fécondant du spermatozoïde a fait l'objet de nombreux travaux.

Sur le plan morphologique, les anomalies de l'acrosome peuvent être responsables d'échecs de fécondation [58]. Le cas le plus extrême est la stérilité définitive des sujets porteurs de spermatozoïdes à tête ronde sans acrosome [59].

En ce qui concerne la relation entre la RA et la fécondation, les premiers travaux, où le taux de RA spontanée était comparé au pourcentage d'ovocytes segmentés en FIV, ont donné des résultats assez contradictoires. Certains auteurs n'ont pas retrouvé une véritable corrélation entre le taux de 
clivage et celui de la RA [51]. D'autres, au contraire, ont relevé qu'un pourcentage anormalement élevé de spermatozoïdes réagis avant tout traitement était souvent lié à une fécondance diminuée $[56,60]$.

En ce qui concerne la relation entre la RA induite et la fécondance, les résultats des études montrent que les échecs de fécondation sont fréquemment liés à une incapacité des spermatozoïdes à réagir en présence d'un inducteur. Ainsi Calvo et coll [61] ont rapporté une réponse diminuée des spermatozoïdes à la stimulation, dans la plupart des stérilités inexpliquées. Cette observation a été également faite par Wolf [39], Fénichel et coll. [56] et Takahashi et coll. [62]. Nous mêmes, nous avons constaté une réponse insuffisante des spermatozoïdes à l'ionophore chez les sujets asthénozoospermiques inféconds [55].

\section{CONCLUSION}

L'appréciation de statut acrosomique représente une approche fonctionnelle intéressante du pouvoir fécondant du spermatozoïde. L'approche dynamique en présence d'un inducteur, même non-physiologique, semble plus appropriée pour l'étude des corrélations entre les 2 fonctions du gamète mâle : RA et fécondation. Toutefois, il existe une telle diversité dans l'utilisation des milieux, des inducteurs et des moyens de détection qu'il est difficile de comparer des résultats et d'évaluer la valeur pronostique d'une telle exploration. D'autre part, il faudrait des études systématiques pour apprécier l'importance de la pathologie touchant cette structure du spermatozoïde.

Il est très souhaitable de standardiser le mode et les milieux de préparation des spermatozoïdes, les protocoles d'induction, la technique d'évaluation du statut acrosomique (méthode simple, reproductible, fiable), pour pouvoir comparer les résultats d'études multicentriques et confirmer que cet aspect de la physiologie du spermatozoïde a une réelle signification clinique.

\section{BIBLIOGRAPHIE}

1. AUSTIN CR. : Observations on the penetration of sperm into the mammalian egg. Aust. J. Sci. Res., 1951, B.4 : 581-596.

2. CHANG MC. : Fertilizing capacity of sperm, deposited in the fallopian tubes. Nature, 1951, $168: 697$ 698.

3. LANGLAIS J, ROBERTS KD. : A molecular membrane model of sperm capacitation and the acrosome reaction of mammalian spermatozoa. Gam. Res. 1985, $12: 183-224$.

4. TESARIK J. From the cellular to the molecular dimension : the actual challenge for human fertilization research. Gam. Res. 1986, $13:$ 47-89.

5. KOEHLER JK. : Surface alteration during the capacitation of mammalian spermatozoa. Am. J. Primatol. 1981 : 131-141.

6. AGUAS AP, PINTO da SILVA P. : Regionalisation of transmembrane glycoproteins in the plasma membrane of boar sperm head is revealed by fracture-label. J. Cell. Biol. 1983, 97 : 1356-1364.

7. AGUAS AP, PINTO da SILVA P. : High density of transmembrane glycoproteins on the flagellar surface of boar sperm cells. J. Cell. Biol.1984, $99: 655-660$.

8. KOEHLER JK. : Human sperm head ultra-structure : A freeze- etching study. J. Ultrastruct. Res. 1972, $39: 520-539$.

9. TESARIK J. : Topographical relationship of intramembrane particle distribution patterns in human sperm membranes. J. Ultrastruct. Res. 1984, 89: 42-55.

10. KOEHLER JK, De CURTIS I, STENCHEVER MA, SMITH D. : Interaction of human sperm with zonafree hamster eggs. Gamete Res. 1982, 6 : 371-386.

11. DAVIS BK. : Timing of fertilization in mammals : Sperm cholesterol/phospholipid ratio as a determinant of the capacitation interval. Proc. Natl. Acad. Sci. USA, 1981, $78: 7560-7564$.

12. DAVIS BK. : Uterine fluid proteins bind sperm cholesterol during capacitation in the rabbit. Experientia, 1982, 38 : 1063-1064.

13. AITKEN RJ, WANG YF, LUI J, BEST F, RICHARDSON DW. : The influence of medium composition, osmolarity and albumin content on the acrosome reaction and fertilizing capacity of human spermatozoa : development of an improved zona-free hamster egg penetration test. Int. J. Androl. 1983, 6,: 180-193.

14. HANDROW RR, LENZ RW, AX R.L. : Structural comparaisons among glycosaninoglycans to promote an acrosome reaction in bovine spermatozoa. Biochem. Biophys. Res. Comm. 1982, 107: 1326-1332. 
15. LENZ RW, BALL GD, LOHSE JK, FIRST NL. AX R.L. : Chondrointin sulfate facilitates an acrosome reaction in bovine spermatozoa as evidenced by light microscopy, electron microscopy and in vitro fertilization. Bio. Reprod. 1983, 28: 683-690.

16. DAVIS BK, BYRNE R, BEDIGIAN K. : Studies on the mechanism of capacitation : albumin mediated changes in plasma membrane lipids during in vitro incubation of rat cells. Proc. Natl. Acad. Sci. USA, 1980, 77: 1546-1550.

17. ROLDAN ERS, HARRISON RAP. : Molecular mechanisms leading to exocytosis during the sperm acrosome reaction. Fertilization in Mammals. Eds Bavister BD. Cummins JM. et Roland ERP. Plenum Press, New-York, 1990 : 179-196.

18. YANAGIMACHI R. : Mammalian fertilization. In "The physiology of reproduction" edited by Knobil E. Neill J. Raven Press, Ltd New-York, 1988 : 135.

19. NILES WD, COHEN FS. : Vesicle fusion with planar membranes as a model system of exocytosis. Ann. NY Acad. Sci. 1991 36: 182-190.

20. LANGLAIS J,, PLANTE L, BLEAU G, CHAPDELAINE A, ROBERTS KD. : Metabolism of lysophosphatidylcholine in relation to sperm capacitation. Fertil. Steril. 1982, 38: 135-136.

21. KOPF GS. : Regulation of sperm function by guanine nucleotide-binding regulatory proteins (G-proteins). In "Meiotoic Inhibition : Molecular Control of Meiosis" Hazeltine, F.P. and First, F.L. (eds),. A.R. Liss, New York, 1988, 357-386.

22. ROLDAN E.R.S., HARRISON RAP. : Absence of active protein kinase $\mathrm{C}$ in ram spermatozoa. Biochem. Biophys. Res Commun.1989, 155 : 901-906.

23. ANDERSON RA, FEATHERGILL KA, ZANEVELD LJD. : Evidence for modulation of the human sperm acrosome reaction by G-proteins. J. Androl. Suppl. 1991, 43.

24. BLACKMORE PF, BEEHE SJ, DANFORTH DR ALEXANDER NJ. : Progesterone and 17 -hydroxyprogesterone. Novel stimulators of calcium influx in human sperm. J. Biol. Chem. 1990, 264 : 1376-1380.

25. SALING PM, STOREY BT. : Mouse gamete interactions during fertilization in vitro. Chlortetracycline as a fluorescent probe for the mouse acrosome raction. J. Cell. Biol. 1979, 83 : 544-552.

26. CROZET N, DUMONT M. : The site of the acrosome reaction during in vivo penetration of the sheep oocyte. Gamete Res. 1984, $10: 97-102$.

27. CROZET N. : Ultrastrucural aspects of in vivo fertilization in the cow. Gamete Res. 1984, $10: 241-247$

28. WASSARMAN PM. : Early events in mammalian fertilization. Ann. Rev. Cell. Biol. 1987, 3 : 109. 115 .
29. RUSSELL L, PETERSON RN, FREUND M. : Morphologic characteristics of chemically induced acrosome reaction in human spermatozoa. Fertil. Steril. $1979,32,87-92$.

30. SALING PM, STOREY BT. : Mouse gamete interactions during fertilization in vitro, chlortetracycline as a fluorescent probe for the mouse sperm acrosome reaction. J. Cell. Biol. 1979, 83 : 544555.

31. LEE MA, TRUCCO GS, BECHTOL KB, WUMMER N, KOPF GS, BLASCO L, STOREY BT. : Capacitation and acrosome reactions in human spermatozoa monitored by a chlortetracylcine fluorescence assay. Fertil. Steril.1987, 48 : 649-658.

32. KOEHLER JK. : Lectins as probes of the spermatozoan surface. Arch. Androl. 1981, 6 : 197-218.

33. TALBOT P, CHACON R.S. : A new procedure for rapidly scoring acrosome reaction of human sperm. Gamete Research. 1980, 3 : 211-216.

34. HOLDEN CA, HYNE RV, SATHANANTHAN AH, TROUNSON AO. : Assessment of human sperm acrosome reaction using concanavalin-A lectin. Molec. Reprod. Develop. 1990, 25 : 247-257.

35. CROSS NL, MORALES P, OVERSTREET JW, HANSON FW. Two simple methods for detecting acrosome-reacted human sperm. Gamete Res. 1986, 15 : 213-226.

36. MENDOZA C, CARRERAS A, MOOS J, TESARIK $J$. Distinction between true acrosome reaction and degenerative acrosome loss by a one step staining method using Pisum sativum agglutin. J.Reprod. Fertil. 1992, 95 : 755-763.

37. KALLAJOKI M, VIRTANEN I, SUOMINEN J. : The fate of acrosomal staining during the acrosome reaction of human spermatozoa as revealed by a monoclonal antibody and PNA-lectin. Int. J. Androl. 1986, 9 : 181-194.

38. MORTIMER D, CURTIS EF, MILLER RG. : Specific labelling by peanut agglutinin of the outer acrosomal membrane of the human spermatozoon. J. Reprod. Fertil. 1987, 81 : 127-135.

39. WOLF DP, BOLDT J, BYRD W, BECHTOL KB. : Acrosomal status evaluation in human ejaculated sperm with monoclonal antibodies. Biol. Reprod. $1986,32: 1157$.

40. FENICHEL P, HSI BL, FARAHIFAR D, DONZEAU M, BARRIER-DELPECH D, YEH CJG. : Evaluation of the human sperm acrosome reaction using a monoclonal antibody, GB24, and fluorescence-activated cell sorter. J. Reprod. Fertil. 1989, $87: 699-706$.

41. TALBOT P, CHACON RS. : A triple-stain technique for evaluating normal acrosome reactions of human sperm. J. Ex. Zool. 1981, 215 : 201-208. 
42. BRAUN J, HIRSCH T, KRAUSE W, ZIEGLER A. : Evaluation of the acrosome reaction using monoclonal antibodies against different acrosomal antigens-comparison with the triple stain techniques. Int. J. Androl. 1991, 14 : 431-436.

43. OKABE M, MATZO S, NAGIRA M, YING X, KOHAMA Y, MIMURA T. : Collection of acrosomereacted human sperm using monoclonal antibodycoated paramagnetic beads. Molecul. Reprod. Dev. 1992, 32 : 389-393.

44. PARINAUD J. VIEITEZ G, LABAL B, RICHOILLEY G. : Selection et micro-injection des spermatozoïdes ayant fait leur réaction acrosomique. Communication aux Journées de Périconceptologie, Toulouse 27-29 Mai 1993.

45. TESARIK J. DRAHORAD J, PEKNICOVA J. : Subcellular immunochemical localisation of acrosin in human spermatozoa during the acrosome reaction and zona pellucida penetration. Fertil. Steril. 1988, $50: 133-140$.

46. DRAHORAD J, CECHOVA D, T'ESARIK J. : Activation of proacrosine by a locally produced component of human follicular fluid. J. Reprod. Fertil. 1988, $83: 599-603$.

47. GOODPASTURE JC, ZAVOS PML, COHEN MR, ZANEVELD LJD. : Relationship of human sperm acrosin and proacrosin to semen parameters. Comparaison between symptomatic men of infertile couples and asymptomatic men, and between different split ejaculates. J. Androl. 1990, 1 : 16-27.

48. MOHSENIAN M, SYNER FN, MOGHISSI KS. : A study of sperm acrosin in patients with unexplained infertility. Fertil. Steril. 1982, $37: 223$ 229.

49. KOUKOULIS GN, VANTMAN D, DENNISON L, BANKS SM, SHERIN RJ. : Low acrosin in a subgroup of men with idiopathic infertility does not correlate with sperm density percent motility, curvilinear velocity of linearity. Fertil. Steril.1989, 52: 120-127.

50. PERREAULT S, ROGERS BJ. : Capacitation pattern of human spermatozozoa. Fertil. Steril. 1982, $38: 258-260$.

51. PLACHOT M, MANIDELBAUM J, JUNCA AM. : Acrosome reaction of human sperm used for in vitro fertilization. Fertil. Steril. 1984, 42 : 418423.

52. PILIKIAN S, MIMOUNI P. : Comparative study of the acrosome reaction and survival of human spermatozoa in various media. Int. J. Androl. 1988, 11 : 465-472.

53. MORTIMER D, CURTIS EF, CAMENZIND AR, TANAKA $S$. : The spontaneous acrosome reaction of human spermatozoa in vitro. Hum. Reprod. $1989,4: 57-62$.
54. OSMAN RA, ANDRIA ML, JONES AD, MEIZEL $\mathrm{S}$. : Steroid induced exocytosis - the human sperm acrosome reaction. Bioch. Biophys. Res. Comm. $1989: 160$.

55. PILIKIAN S, GUERIN JF, ADELEINE P, ECOCIIARD R, CZYBA JC. : Spontaneous and ionophore induced acrosome reaction in asthenozoospermic infertile semen. Hum. Reprod. 1992, 7 : 991-992.

56. FENICHEL P, DONZEAU M, FARAHIFAR D, BASTERIS B, AYRAUD N, HSI BL. : Dynamics of human sperm acrosome reaction, relation with in vitro fertilization. Fertil. Steril. 1991, 55 : 994-999.

57. TESARIK J, MENDOZA C, CARRERAS A. : Fast acrosome reaction measure : a highly sensitive method for evaluating stimulus-induced acrosome. Fertil. Steril. 1993, $59: 424-430$.

58. JEULIN C, FENEUX D, SERRES C, JOUANNET P, GUILLET-ROSSO, BELAISH-ALLART J, FRYDMAN R, TESTARD J. Sperm factors related to failure of human in vitro fertilization. J. Reprod. Fertil. 1986, 76 : 735-741.

59. JEYENDRAN RS, VAN DER VEN HH, KENNEDY WP, HEATH E, PEREZ-PELAEZ M, SOBRERO AJ, ZANEFELD LJD. : Acrosomeless sperm, a cause of primary male infertility. Andrologia, 1985, $17: 31-37$.

60. TOPFER-PEDERSEN E, HEISSLER E, SCHILL WB. : The kinetic of acrosome reaction, an additional sperm parameter. Andrologia, 1985, $17: 24-233$.

61. CALVO L, VANTMAN D, BANKS SM, TEZON J, KOUKOULIS GN, DENNISON L, SHERINS RJ. : Follicular fluid induced acrosome reaction distinguishes a subgroup of men with unexpained infertility not identified by semen analysis. Fertil. Steril. $1989,52: 1048-1054$.

62. TAKAHASHI $\mathrm{K}$, WETZELS MM, GOVERDE HJ, BASTIAANS BA, JANSSEN HJG, ROLLAND R. : The kinetics of the acrosome reaction of human spermatozoa and its correlaiton with in vitro fertilization. Fertil. Steril. 1992, $57: 889-894$.

ABSTRACT

\section{The acrosome reaction. Review of the different methods of evaluation and teh interest of acrosomal investigation}

\author{
S. PILIKIAN
}

\section{Laboratoire de Biologie de la Repro- duction et du Développement, Lyon}

The acrosome reacting capacity of spermatozoa is essential for the fertili- 
zation of the oocyte. Several methods are available for the evaluation of the acrosomal status ; each method detects a step of the reaction which is a continuous process. Due to the diversity and the relative specificity of each technique, it is difficult to compare results in absolute values.

The reaction is Ca++ dependent. The use of $\mathrm{Ca}++$ ionophores is valuable for a dynamic study which gives a better appreciation of the capacity of spermatozoa to react to a specific stimulus.
Many authors have attempted to correlate the acrosomal status to the fertilizing ability of spermatozoa. The results show a reduced induction of $A R$ in hypofertile sperm.

More studies with standard materials and methods are needed in order to confirm the diagnostic value of this test.

Key words : Human spermatozoa, acrosome raction tests, acrosome reaction - in vitro fertilization. 